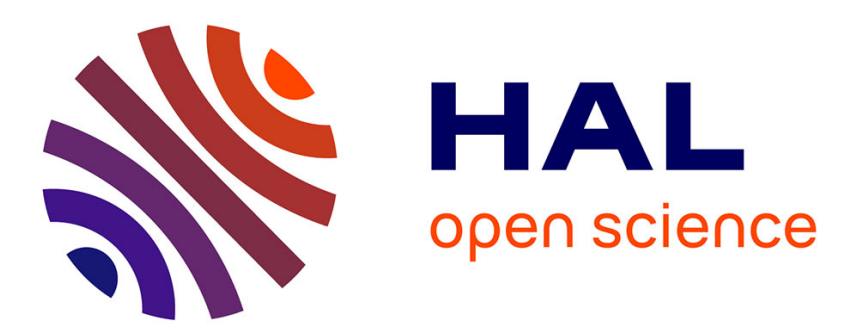

\title{
Retrieving surface waves from ambient seismic noise using seismic interferometry by multidimensional deconvolution
}

Karel N. van Dalen, T. Dylan Mikesell, Elmer N. Ruigrok, K. Wapenaar

\section{To cite this version:}

Karel N. van Dalen, T. Dylan Mikesell, Elmer N. Ruigrok, K. Wapenaar. Retrieving surface waves from ambient seismic noise using seismic interferometry by multidimensional deconvolution. Journal of Geophysical Research: Solid Earth, 2015, 120 (2), pp.944 - 961. 10.1002/2014JB011262 . hal01382583

\section{HAL Id: hal-01382583 \\ https://hal.science/hal-01382583}

Submitted on 8 Oct 2021

HAL is a multi-disciplinary open access archive for the deposit and dissemination of scientific research documents, whether they are published or not. The documents may come from teaching and research institutions in France or abroad, or from public or private research centers.
L'archive ouverte pluridisciplinaire HAL, est destinée au dépôt et à la diffusion de documents scientifiques de niveau recherche, publiés ou non, émanant des établissements d'enseignement et de recherche français ou étrangers, des laboratoires publics ou privés. 


\section{Journal of Geophysical Research: Solid Earth}

\author{
RESEARCH ARTICLE \\ 10.1002/2014JB011262 \\ Key Points: \\ - Use multidimensional deconvolution \\ to retrieve surface waves from \\ ambient noise \\ - Phase corrections and removal of $f, k$ \\ notch compared to cross-correlation \\ result \\ - Higher signal-to-noise ratio in MDD \\ result compared to cross-correlation \\ result
}

Supporting Information:

- Readme

- Figure S1

Correspondence to:

K. N. van Dalen,

k.n.vandalen@tudelft.n

Citation:

van Dalen, K. N., T. D. Mikesell,

E. N. Ruigrok, and K. Wapenaar

(2015), Retrieving surface waves from ambient seismic noise using seismic interferometry by multidimensional deconvolution, J. Geophys. Res. Solid Earth, 120, 944-961, doi:10.1002/2014JB011262.

Received 9 MAY 2014

Accepted 11 DEC 2014

Accepted article online 17 DEC 2014

Published online 6 FEB 2015

\section{Retrieving surface waves from ambient seismic noise using seismic interferometry by multidimensional deconvolution}

\author{
Karel N. van Dalen ${ }^{1}$, T. Dylan Mikesell ${ }^{2,3}$, Elmer N. Ruigrok ${ }^{1}$, and Kees Wapenaar ${ }^{1}$ \\ ${ }^{1}$ Department of Geoscience and Engineering, Delft University of Technology, Delft, Netherlands, ${ }^{2}$ Department of Earth, \\ Atmospheric and Planetary Sciences, Massachusetts Institute of Technology, Cambridge, Massachusetts, USA, ${ }^{3}$ Previously \\ at Geoazur, Université de Nice Sophia-Antipolis/CNRS/IRD, Nice, France
}

Abstract Retrieving virtual source surface waves from ambient seismic noise by cross correlation assumes, among others, that the noise field is equipartitioned and the medium is lossless. Violation of these assumptions reduces the accuracy of the retrieved waves. A point-spread function computed from the same ambient noise quantifies the associated virtual source's spatial and temporal smearing. Multidimensional deconvolution (MDD) of the retrieved surface waves by this function has been shown to improve the virtual source's focusing and the accuracy of the retrieved waves using synthetic data. We tested MDD on data recorded during the Batholiths experiment, a passive deployment of broadband seismic sensors in British Columbia, Canada. The array consisted of two approximately linear station lines. Using 4 months of recordings, we retrieved fundamental-mode Rayleigh waves $(0.05-0.27 \mathrm{~Hz})$. We only used noise time windows dominated by waves that traverse the northern line before reaching the southern ( $2.5 \%$ of all data). Compared to the conventional cross-correlation result based on this subset, the MDD waveforms are better localized and have significantly higher signal-to-noise ratio. Furthermore, MDD corrects the phase, and the spatial deconvolution fills in a spectral ( $f, k$ domain) gap between the single-frequency and double-frequency microseism bands. Frequency whitening of the noise also fills the gap in the cross-correlation result, but the signal-to-noise ratio of the MDD result remains higher. Comparison of the extracted phase velocities shows some differences between the methods, also when all data are included in the conventional cross correlation.

\section{Introduction}

Seismic interferometry is a technique that uses wavefield recordings to create virtual seismic sources at locations where only receivers are present. By cross-correlating observations at two different seismic receivers, one retrieves an approximation of the Green's function as if one of the receivers were a source: the virtual source response [e.g., Campillo and Paul, 2003; Larose et al., 2006; Wapenaar and Fokkema, 2006; Schuster, 2009]. This technique has been used in many different applications and scales, ranging from ultrasonics to seismology [cf. Weaver and Lobkis, 2006].

Surface wave retrieval has received wide attention, because wavefield recordings are often dominated by surface waves. In regional seismology, virtual source surface wave responses are retrieved by applying seismic interferometry to recorded microseisms generated by oceanic waves coupled to the seafloor, thus creating seismic waves in the solid Earth [Shapiro and Campillo, 2004; Shapiro et al., 2005; Sabra et al., 2005a; Bromirski et al., 2013], or to recorded earthquakes by exploiting the seismic coda [Campillo and Paul, 2003]. Although it follows from the theory that the full Green's function can be obtained [Wapenaar, 2004; Sánchez-Sesma and Campillo, 2006; Sánchez-Sesma et al., 2006; Halliday and Curtis, 2008], often only the fundamental-mode Rayleigh wave is retrieved due to the specific microseism energy generation [Shapiro and Campillo, 2004; Sabra et al., 2005a]. Retrieved surface waves have been successfully used to determine velocity images of the Earth's crust [e.g., Sabra et al., 2005a, 2005b; Shapiro et al., 2005; Gerstoft et al., 2006; Yang et al., 2008].

Using theoretical arguments, it can be verified that retrieving Green's functions by cross correlation relies on the medium being lossless and the noise field being equipartitioned [Wapenaar and Fokkema, 2006; Sánchez-Sesma and Campillo, 2006]; the latter condition implies that the receivers whose observations are cross correlated are uniformly illuminated from all directions. This can be achieved when the noise sources are regularly distributed around the receivers, the sources are mutually uncorrelated, and their power 
spectra are identical. Due to these conditions being only partially fulfilled in many situations [e.g., Mulargia, 2012], the accuracy of the retrieved surface waves can be limited; the virtual source responses are often blurred by artifacts (i.e., spurious events and errors in relative amplitude and phase). It is clear that this may lead to errors in the estimated dispersion curves and, consequently, in the velocity profiles obtained by tomographic inversion [e.g., Tsai, 2009].

Several methods have been proposed in order to overcome the relatively strict conditions associated with the cross-correlation (CC) method and correct for the corresponding artifacts. Among them are the techniques of spatiotemporal inverse filtering [Berkhout, 1982; Tanter et al., 2001; Gallot et al., 2012] and of seismic interferometry by multidimensional deconvolution (abbreviated to MDD) [Wapenaar and van der Neut, 2010] that are applicable both for sequential controlled sources and for noise sources. The latter technique is based on the reciprocity theorem of the convolution type rather than on that of the correlation type. Two advantages of MDD are that it does not rely on the medium being lossless and can overcome irregularities in the illumination from the noise sources [Wapenaar et al., 2011b]. In addition, MDD allows for differences in source power spectra and source types as well as some degree of correlation between the sources, as opposed to the cross-correlation (CC) method. In heursitic terms, MDD introduces an additional processing step in which the cross-correlation function (CCF) is deconvolved (spatially and temporally) by a point-spread function (PSF), which is calculated from the same noise fields. This PSF captures the irregularity of the source distribution as well as effects due to anelasticity and correlated sources. The PSF quantifies the associated smearing of the virtual source in space and time, and deconvolving by the PSF can correct for this smearing. The virtual source then becomes better focused and, accordingly, the retrieved virtual source response more accurate.

Using a numerical experiment to test MDD, Wapenaar et al. [2011a] showed that artifacts in retrieved fundamental-mode surface waves can be suppressed and the estimated phase velocities become more accurate. However, MDD has not yet been applied to real ambient noise surface wave observations. Therefore, we tested MDD on data recorded during the Batholiths experiment [Calkins et al., 2010], which was a passive deployment of broadband seismic sensors in British Columbia (Canada; 2005-2006) with an array geometry fit for applying MDD (see Figure 1); the recorded noise is dominated by microseisms. In this paper, we report on the results and investigate whether the accuracy of the retrieved responses and estimated phase velocities indeed increases. We also discuss the estimation of the surface wave attenuation coefficient, which should be possible as the losses are correctly taken into account in the MDD scheme [cf. Wapenaar et al., 2010].

\section{Seismic Interferometry by CC and MDD}

In this section, we briefly review the theory of seismic interferometry by MDD in order to highlight the differences with the more conventional CC method. We restrict the discussion to scalar fields because, at the free surface of a layered medium, a single-mode dispersive surface wave behaves in the far field very similar to a scalar body wave in a 2-D homogeneous lossy medium [e.g., Tsai, 2011]. The space-frequency domain representations of both waves can be written in terms of the zeroth-order Hankel function of the second kind (when an $\exp (\mathrm{i} \omega t)$ dependence is adopted in the inverse Fourier transform): $A(\theta, \omega) H_{0}^{(2)}(k r)$, where $k=k(\omega)$ is the complex-valued wave number, $r$ is the propagation distance, and $A(\theta, \omega)$ is a complex coefficient depending on frequency $\omega$ and direction of radiation $\theta$ (measured from the source). In both cases, the propagation and the geometrical and intrinsic amplitude decays are described by the Hankel function. The coefficient is source specific and generally different for surface waves and 2-D scalar waves, as different sources result in different radiation patterns. For surface waves, the coefficient also depends on the vertical coordinates of source and receiver, but these are left out for brevity; we restrict to observations at one depth level (the free surface), and different source depths just imply different values of the coefficient.

\subsection{Theory}

According to the conventional CC method, bandlimited versions of the virtual source Green's function $\hat{G}\left(\mathbf{x}_{R}, \mathbf{x}\right)$ and its time reverse $\hat{G}^{*}\left(\mathbf{x}_{R}, \mathbf{x}\right)$ can be retrieved by cross-correlating observations at two different stations $\mathbf{x}_{R}$ and $\mathbf{x}$ due to bandlimited point sources located at the boundary $S$ that surrounds the two 

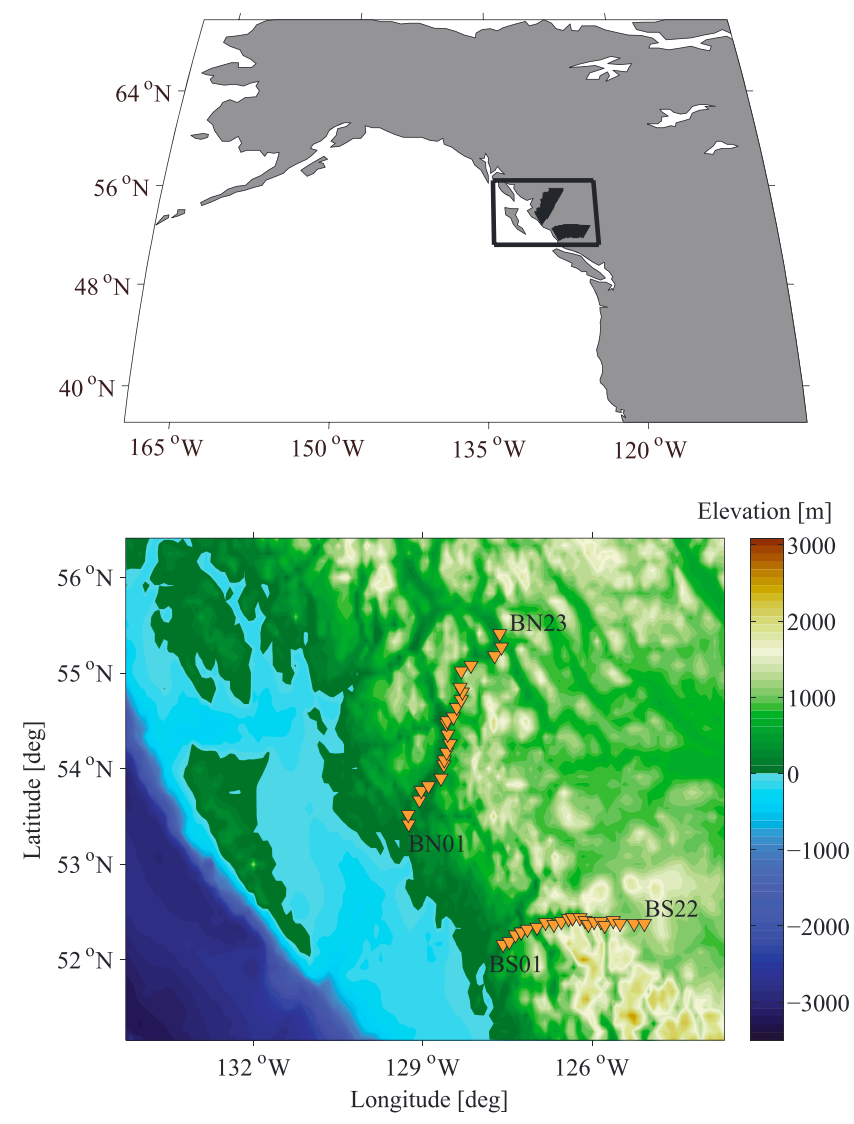

Figure 1. Batholiths experiment in British Columbia (Canada; 2005-2006): orientation with respect to (top) the coastline and (bottom) zoom of framed area, where BN denotes the north seismic array and BS the south array. The specific stations names are given in Tables 1 and 2.

receivers (after equation (36) of Wapenaar and Fokkema [2006]):

$$
\begin{aligned}
\left(\hat{G}\left(\mathbf{x}_{R}, \mathbf{x}\right)+\hat{G}^{*}\left(\mathbf{x}_{R}, \mathbf{x}\right)\right) \hat{P} & \sim \oint_{S} \hat{u}\left(\mathbf{x}_{R}, \mathbf{x}_{S}\right) \hat{u}^{*}\left(\mathbf{x}, \mathbf{x}_{S}\right) \mathrm{d} S \\
& \sim \sum_{j} \hat{u}\left(\mathbf{x}_{R}, \mathbf{x}_{S}^{(j)}\right) \hat{u}^{*}\left(\mathbf{x}, \mathbf{x}_{S}^{(j)}\right),
\end{aligned}
$$

where $\mathbf{x}_{S}$ and $\mathbf{x}_{S}^{(j)}$ indicate the source locations in the continuous and discrete representations, respectively; see also Figure 2 (top), where $D$ is the domain that contains the two stations and is enclosed by $S$. The hats in equation (1) refer to the frequency $(\omega)$ domain, the $*$ in the superscript implies taking the complex conjugate (which relates to time reversal, so that the time domain version of equation (1) involves cross correlations), and $\hat{P}=\hat{P}(\omega)$ is the source power spectrum that is assumed the same for all sources. In our context, $u$ denotes a displacement component (i.e., a scalar; $G$ denotes the associated Green's function) recording a single-mode surface wave at the free surface of a layered medium [e.g., Tsai, 2011]. Furthermore, the observations are defined as

$$
\hat{u}\left(\mathbf{x}_{R}, \mathbf{x}_{S}^{(j)}\right)=\hat{G}\left(\mathbf{x}_{R}, \mathbf{x}_{S}^{(j)}\right) \hat{s}^{(j)}
$$

and $\hat{u}\left(\mathbf{x}, \mathbf{x}_{S}^{(j)}\right)$ defined similarly; here $\hat{s}^{(j)}=\hat{s}^{(j)}(\omega)$ signifies the source spectrum of a specific source (where, $\left.\hat{P}=\left|\hat{S}^{(j)}\right|^{2}\right)$. When only the gray sources shown in Figure 2 are present and the illumination is one-sided, only the virtual source response $\hat{G}\left(\mathbf{x}_{R}, \mathbf{x}\right) \hat{P}$ (i.e., the causal part of the full signal) is retrieved.

In principle, equation (1) requires that the responses due to the individual sources are known. In situations where ambient noise is employed, only the observations due to simultaneously acting sources are known:

$$
\hat{u}\left(\mathbf{x}_{R}\right)=\sum_{j} \hat{u}\left(\mathbf{x}_{R}, \mathbf{x}_{S}^{(j)}\right)=\sum_{j} \hat{G}\left(\mathbf{x}_{R}, \mathbf{x}_{S}^{(j)}\right) \hat{s}^{(j)}
$$



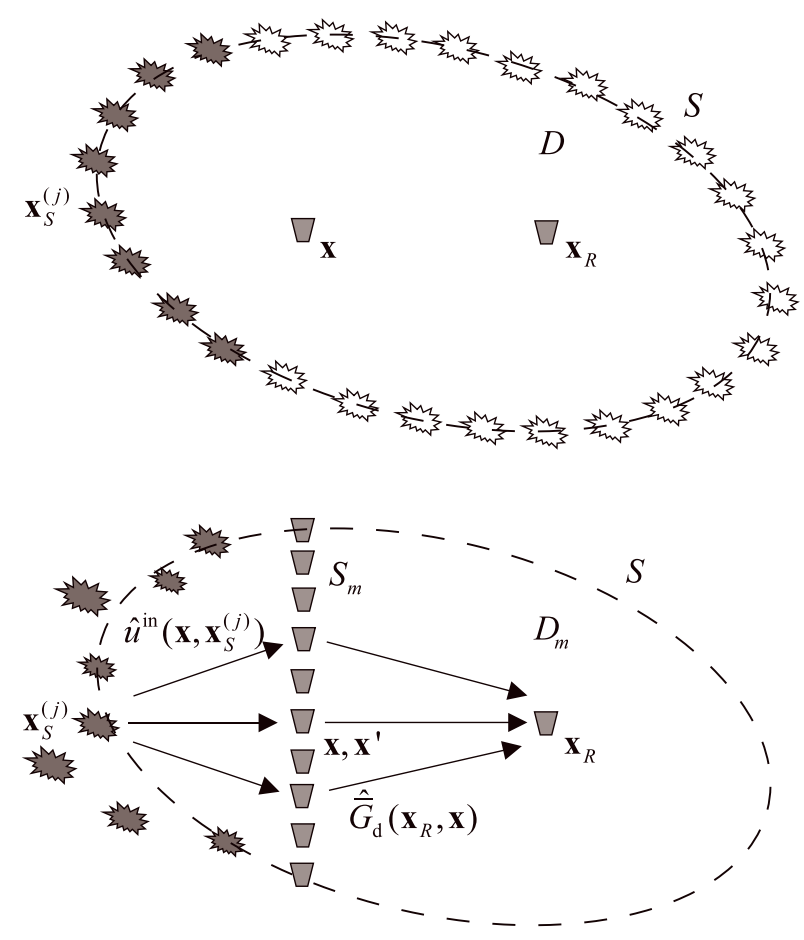

Figure 2. Configurations associated with seismic interferometry (top) by cross correlation and (bottom) by multidimensional deconvolution. Symbols are defined in the text.

and $\hat{u}(\mathbf{x})$ defined similarly. However, the virtual source response can still be retrieved by taking the ensemble average $\langle\cdot\rangle$ of the cross correlation of the observations. Assuming one-sided illumination, we can write

$$
\left\langle\hat{u}\left(\mathbf{x}_{R}\right) \hat{u}^{*}(\mathbf{x})\right\rangle \sim \hat{G}\left(\mathbf{x}_{R}, \mathbf{x}\right) \hat{P},
$$

provided that the noise sources are mutually uncorrelated and have the same power spectrum:

$$
\left\langle\hat{s}^{(i)} \hat{s}^{(j) *}\right\rangle=\delta_{i j} \hat{P}
$$

(note that the cross-correlated observations in equation (4) involve different summation indices $i$ and $j$ ). This is clarified by substituting expressions of $\hat{u}\left(\mathbf{x}_{R}\right)$ and $\hat{u}(\mathbf{x})$ (equation (3)) into equation (4) and using the condition expressed in equation (5); we then obtain the causal part of equation (1). In practice, the ensemble average in equation (4) is replaced by integrating over sufficiently long time and/or averaging over different time intervals. Apart from the condition in equation (5), the virtual source response is retrieved successfully provided that the sources are regularly distributed along the boundary $S$ (cf. equation (1)), the medium is lossless, and all sources are the same (i.e., the same radiation pattern and magnitude).

\subsection{MDD Theory}

While the CC method is based on the reciprocity theorem of the correlation type, the MDD method is derived from the reciprocity theorem of the convolution type [Wapenaar et al., 2011b]. For the configuration shown in Figure 2 (bottom), where the medium $D_{m}$ to the right of boundary $S_{m}$ is assumed to be an half-space (and $S_{m}$ thus has infinite extent), the derivation results in the following expression for the forward propagation of the wavefield excited at a specific source $\mathbf{x}_{S}^{(j)}$ via $\mathbf{x}$, located at $S_{m}$, to receiver $\mathbf{x}_{R}$ :

$$
\hat{u}\left(\mathbf{x}_{R}, \mathbf{x}_{\mathrm{S}}^{(j)}\right)=\int_{S_{m}} \hat{\bar{G}}_{\mathrm{d}}\left(\mathbf{x}_{R}, \mathbf{x}\right) \hat{u}^{\mathrm{in}}\left(\mathbf{x}, \mathbf{x}_{\mathrm{S}}^{(j)}\right) \mathrm{d} S,
$$

where $\hat{u}^{\text {in }}$ denotes the inward propagating wavefield at $S_{m}$ into $D_{m}$, and $\hat{\bar{G}}_{\mathrm{d}}\left(\mathbf{x}_{R}, \mathbf{x}\right)$ is a dipole Green's function that propagates the wavefield from stations $\mathbf{x}$ to $\mathbf{x}_{R}$, as indicated in Figure 2 . The overbar indicates that the propagator is defined in a reference medium that is different from the true one in the sense that it is free of scatterers left of $S_{m}$, but it is the same as the true medium inside $D_{m}$. Contrary to equation (1), equation (6) also holds for media with losses. 
Equation (6) holds for each source $j$, but the associated individual responses are not known in the situation of simultaneous noise sources. Therefore, we sum all the equations, so that we obtain an expression in terms of the total observations:

$$
\hat{u}\left(\mathbf{x}_{R}\right)=\int_{S_{m}} \hat{\bar{G}}_{\mathrm{d}}\left(\mathbf{x}_{R}, \mathbf{x}\right) \hat{u}^{\mathrm{in}}(\mathbf{x}) \mathrm{d} S,
$$

where $\hat{u}\left(\mathbf{x}_{R}\right)$ and $\hat{u}^{\text {in }}(\mathbf{x})$ are defined similarly as in equation (3). MDD involves solving equation (7) for $\hat{\bar{G}}_{\mathrm{d}}\left(\mathbf{x}_{R}, \mathbf{x}\right)$. To this end, we first cross correlate the left- and right-hand sides with the inward propagating field observed at $S_{m}$ and take the ensemble average. Introducing an auxiliary coordinate $\mathbf{x}^{\prime}$ at $S_{m}$ to distinguish between the different stations whose observations are cross correlated, we obtain

$$
\hat{C}\left(\mathbf{x}_{R}, \mathbf{x}^{\prime}\right)=\int_{S_{m}} \hat{\bar{G}}_{\mathrm{d}}\left(\mathbf{x}_{R}, \mathbf{x}\right) \hat{\Gamma}\left(\mathbf{x}, \mathbf{x}^{\prime}\right) \mathrm{d} S,
$$

where $\hat{C}\left(\mathbf{x}_{R}, \mathbf{x}^{\prime}\right)$ and $\hat{\Gamma}\left(\mathbf{x}, \mathbf{x}^{\prime}\right)$ are the cross-correlation function (CCF) and the point-spread function (PSF), respectively, defined as

$$
\begin{aligned}
\hat{C}\left(\mathbf{x}_{R}, \mathbf{x}^{\prime}\right) & =\left\langle\hat{u}\left(\mathbf{x}_{R}\right) \hat{u}^{\text {in* }}\left(\mathbf{x}^{\prime}\right)\right\rangle, \\
\hat{\Gamma}\left(\mathbf{x}, \mathbf{x}^{\prime}\right) & =\left\langle\hat{u}^{\text {in }}(\mathbf{x}) \hat{u}^{\text {in } *}\left(\mathbf{x}^{\prime}\right)\right\rangle .
\end{aligned}
$$

The associated cross-correlations $\hat{P}^{(i j)}$ of the noise sources can be defined as

$$
\left\langle\hat{s}^{(i)} \hat{s}^{(j) *}\right\rangle=\hat{P}^{(i j)}
$$

MDD solves the virtual source response $\hat{\bar{G}}_{\mathrm{d}}\left(\mathbf{x}_{R}, \mathbf{x}\right)$ from equation (8). As shown below, this is achieved by discretizing equation (8) and solving the resulting system of equations by least squares inversion. Before going into detail, we first discuss the nature of this system, which depends on the conditioning of the PSF. In situations where the noise sources are mutually uncorrelated (as in the CC method), we have

$$
\left\langle\hat{s}^{(i)} \hat{s}^{(j) *}\right\rangle=\delta_{i j} \hat{P}^{(j)}
$$

where $\hat{P}^{(j)}$ is the power spectrum of a specific source, simplifying the CCF and PSF to

$$
\begin{aligned}
\hat{C}\left(\mathbf{x}_{R}, \mathbf{x}^{\prime}\right) & =\sum_{j} \hat{u}\left(\mathbf{x}_{R}, \mathbf{x}_{S}^{(j)}\right) \hat{u}^{\text {in* }}\left(\mathbf{x}^{\prime}, \mathbf{x}_{S}^{(j)}\right), \\
\hat{\Gamma}\left(\mathbf{x}, \mathbf{x}^{\prime}\right) & =\sum_{j} \hat{u}^{\text {in }}\left(\mathbf{x}, \mathbf{x}_{S}^{(j)}\right) \hat{u}^{\text {in } *}\left(\mathbf{x}^{\prime}, \mathbf{x}_{S}^{(j)}\right) .
\end{aligned}
$$

These are the CCF and PSF defined for the situation of sequential sources [van der Neut et al., 2010; Wapenaar et al., 2011b], where the responses can be measured separately. Solving equation (8) by MDD is thus essentially the same for sequential sources and for uncorrelated noise sources. When the noise sources are not mutually uncorrelated (equation (11)), the resulting system of equations can be underdetermined, leading to difficulties in the inversion [Wapenaar et al., 2012]. In practice, the sources are not fully correlated (equation (11)) nor fully uncorrelated (equation (12)), but somewhere in between. Solving equation (8) by MDD does not require that the sources are fully uncorrelated. However, the better equation (12) is satisfied, the more stable the inversion of equation (8) can be carried out; the limits of the physical conditions under which stable inversion is still possible need further investigation [Wapenaar et al., 2012].

In order to solve the virtual source response $\hat{\bar{G}}_{\mathrm{d}}\left(\mathbf{x}_{R}, \mathbf{x}\right)$ from equation (8), the CCF and PSF should be computed from the ambient noise observations, similar to the CC method. The major difference is that more stations are needed in order to compute the PSF (cf. Figure 2). We emphasize the resemblance of the CCF (equation (9)) with the left-hand side of equation (4); given that the illumination is one-sided, results obtained with both functions are very similar. Equation (8) now states that the conventional CC result is proportional to the sought-for Green's function convolved in space and time with a source function being the PSF (for a horizontally varying medium, it is more appropriate to speak of a space-variant spatial convolution), which quantifies the spatial and temporal blurring of the virtual source (of the CC result) centered at $\mathbf{x}^{\prime}$. The PSF is smeared in time and space depending on the illumination, the noise sources 
being correlated, and the level of the losses in the medium (see also section 4). The more ideal the conditions (i.e., regular illumination, uncorrelated sources, and no losses), the more focused the virtual source will be, and the higher the quality of the CCF. Deconvolving the CCF by the PSF corrects for the imprint of the virtual source and yields a more accurate and cleaner virtual source response given enough aperture at $S_{m}$, the sampling conditions at $S_{m}$ are fulfilled, and the sources are not correlated too significantly. Artifacts are then suppressed, the signal-to-noise ratio (SNR) is improved, and estimated surface wave phase velocities become more accurate [Wapenaar et al., 2011a].

To perform MDD in practical situations, equation (8) is discretized along $S_{m}$, as indicated before. For each frequency, a system of equations is obtained that can be written in matrix form [Wapenaar et al., 2011a, 2011b]:

$$
\hat{\mathbf{C}}=\hat{\mathbf{G}}_{\mathrm{d}} \hat{\boldsymbol{\Gamma}}
$$

where the rows and columns of $\hat{\mathbf{C}}$ relate to different $\mathbf{x}_{R}$ and $\mathbf{x}^{\prime}$ stations, respectively, and the matrices $\hat{\mathbf{G}}_{\mathrm{d}}$ and $\hat{\boldsymbol{\Gamma}}$ are organized similarly. The virtual source response can be obtained by right multiplying with the stabilized inverse of $\hat{\Gamma}$ :

$$
\hat{\mathbf{G}}_{\mathrm{d}} \cong \hat{\mathbf{C}}\left(\hat{\boldsymbol{\Gamma}}+\varepsilon^{2} \mathbf{I}\right)^{-1},
$$

where $\varepsilon^{2}$ is a small stabilization constant and $\mathbf{I}$ is the unity matrix. The following matrix,

$$
\hat{\mathbf{Y}}=\hat{\boldsymbol{\Gamma}}\left(\hat{\boldsymbol{\Gamma}}+\varepsilon^{2} \mathbf{I}\right)^{-1},
$$

can be used to diagnose the quality of the MDD. For a perfect deconvolution, the matrix should be an identity matrix, implying that the associated space-time domain function $\Upsilon\left(\mathbf{x}, \mathbf{x}^{\prime}, t\right)$ is a bandlimited spike. We refer to $Y$ as the virtual source function, as its distribution represents the source function of the MDD-retrieved virtual source response.

We note that, apart from being able to handle losses and irregularities in the illumination, MDD is also more general with respect to the properties of individual sources. Even in the case of mutually uncorrelated noise sources (equation (12)), the power spectra $\hat{P}^{(j)}$ of different sources can differ (indicated by the sources of varying magnitude in Figure 2), which is not the case in the $\mathrm{CC}$ method. In addition, differences in source types (i.e., their directivities) are allowed, which can be seen from equation (6); by taking derivatives with respect to $\mathbf{x}_{S}^{(j)}$ in the left- and right-hand sides, the propagator, which is retrieved using MDD, stays the same, and one just needs the same type of observation at $\mathbf{x}_{R}$ and $\mathbf{x}$ (i.e., particle motion) for different sources. Therefore, the application of MDD to real ambient noise fields is promising.

\section{Data Processing Applied to Batholiths Data}

This paper contains results of MDD applied to data recorded during the Batholiths experiment [Calkins et al., 2010], which was a passive deployment of broadband seismic sensors in British Columbia (Canada; 2005-2006) with an array geometry fit for MDD. As the sensors were located close to the shoreline, the recorded noise is expected to be dominated by microseisms. We analyzed the vertical component of the observed particle motion, which is the same component as usually taken in the conventional CC method [Shapiro and Campillo, 2004]. In this section, we describe the relevant processing steps to prepare the data for the MDD introduced in section 2.

The first step in the continuous-noise data processing was to cut the data into $10 \mathrm{~min}(600 \mathrm{~s}$ ) long traces. All instruments in this survey were the same sensor and digitizer; therefore, we neglected the instrument deconvolution in our data processing. We detrended and demeaned each trace, applied a third-order zero-phase Butterworth band-pass filter from 0.05 to $0.27 \mathrm{~Hz}$ and time normalized the amplitudes following equation (1) from Bensen et al. [2007]. The weighting factor for sample $n$ is

$$
w_{n}=\frac{1}{N} \sum_{k=n-N}^{n+N}\left|d_{k}\right|
$$

where the normalized data are defined as $d_{n}^{\text {norm }}=d_{n} / w_{n}$. We chose a half-window length equal to $1 /\left(4 f_{\min }\right)=5 \mathrm{~s}$ because Bensen et al. [2007] determined an optimal window length of half the 

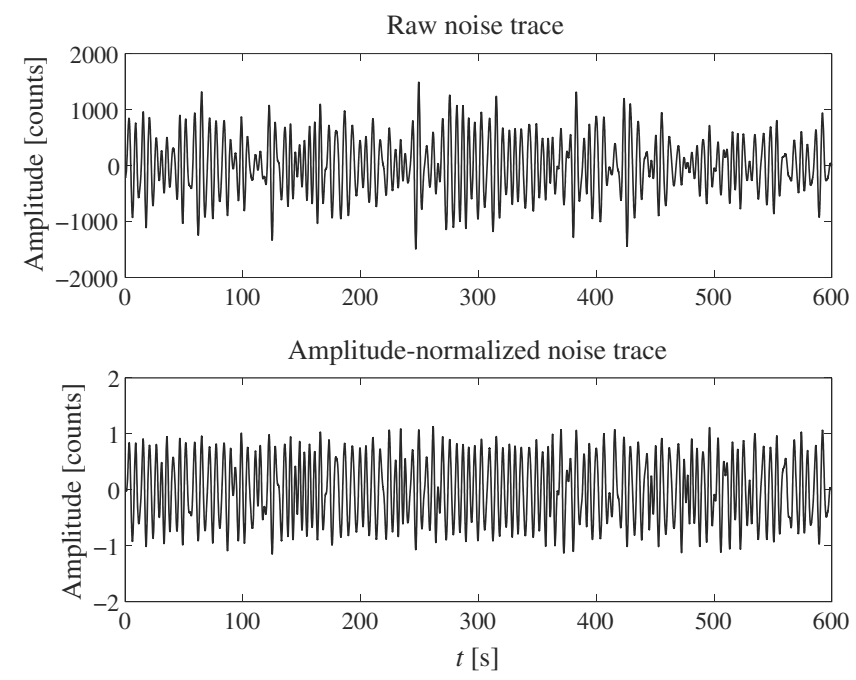

Figure 3. Example of (top) raw noise trace and (bottom) the amplitude-normalized version.

maximum period. The sample interval of the data is $0.025 \mathrm{~s}$, so that $N=200$ samples. After applying this running-absolute-mean normalization, we applied a $5 \mathrm{~s}$ cosine taper to the beginning and end of each trace. An example of a data trace before and after normalization is shown in Figure 3. We did not apply frequency whitening [Bensen et al., 2007]; instead, we let the MDD modify the spectrum post cross correlation.

For the application of MDD, we consider the domain southeast of the BN array to be $D_{m}$ (cf. Figures 1 and 2); obviously, the array that samples the wavefields at $S_{m}$ (i.e., the BN array) has finite extent, which introduces approximations in the MDD. As MDD requires the inward propagating field to compute the CCF and PSF (cf. equations (6), (9), and (10)), only the seismic noise where the dominant source is to the northwest is used, thus providing noise that arrives at the BN array before continuing to the BS array. In order to determine the noise illumination for a given time segment, we performed frequency-wave number analysis [e.g., Lacoss et al., 1969; Rost and Thomas, 2002] on each processed time segment. We used time shifting and stacking over noise traces to estimate the beam power as function of ray parameter and back azimuth. This technique is often referred to as beamforming, and we used the results to find the back azimuth and ray parameter associated with the maximum power of the time segments; note that the orientation of the BN and BS arrays is different, which enables us to determine the direction of the noise. The L-shaped array causes some smearing of the beam power as shown by Rost and Thomas [2002] and van Wijk et al. [2011]. However, in the current case, the smearing does not deteriorate our ability to isolate the maximum power. In Figure S1 in the supporting information, we show the array response at different frequencies for Rayleigh wave velocity coming from an azimuth of $260^{\circ}$. We see that side lobes appear (due to aliasing); however, the maximum is well recovered.

In order to beamform a 10 min group of traces, we required to have data from at least 20 of the 46 stations to ensure that the smearing is not too large. We averaged the beam power over all band-passed frequencies. From here, we found the location of the maximum beam power in each 10 min time window. If the maximum beam power arrived from between 290 and $360^{\circ}$ - from the northwest direction — with a ray parameter between 0.25 and $0.33 \mathrm{~s} / \mathrm{km}$, we kept those data for cross correlation. These ray parameters correspond to a velocity range of 3-4 km/s, which is the range of local Rayleigh wave velocities; Figure 5 in van Wijk et al. [2011] shows that most of the Rayleigh wave noise energy is in the double-frequency microseism band with a phase velocity of $3.1-3.4 \mathrm{~km} / \mathrm{s}$.

The beamforming results are displayed in Figure 4, where we show the stacked power distribution over back azimuth and ray parameter for all data used (bottom) and for the data subset satisfying our ray parameter and back azimuth requirements (top); note that the data have been split into three frequency bands (i.e., the single-frequency (SF) and double-frequency (DF) microseismic bands, and an intermediate (GP) band) to facilitate the analysis in section 4. The direction of maximum beam power in the SF and DF 

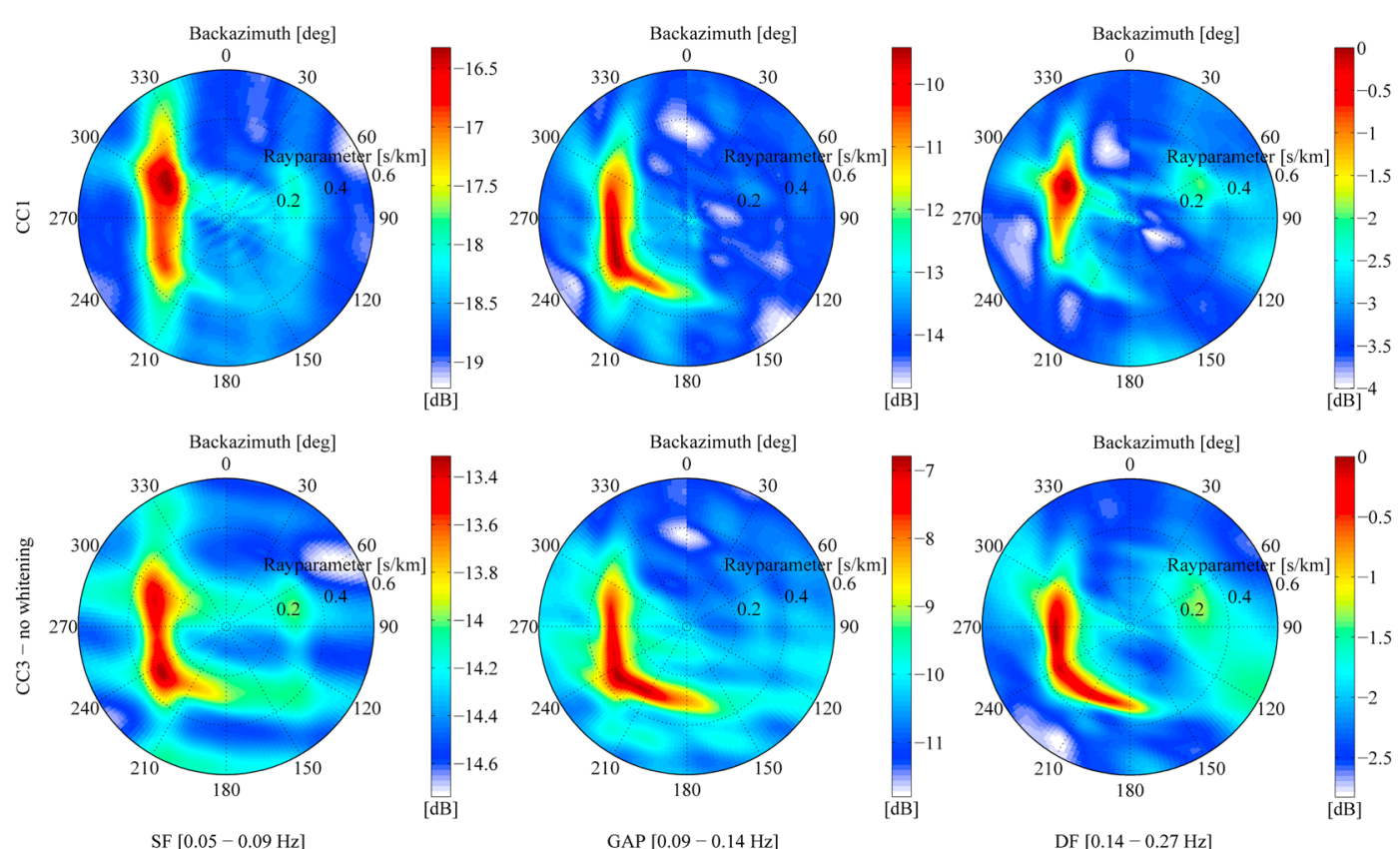

Figure 4. Power distribution over back azimuth and ray parameter (top) of the data subset selected using beamforming and (bottom) of the complete 4 month data set, split into three different frequency bands: single-frequency (SF) and double-frequency (DF) microseismic bands, and intermediate (GP) band. All amplitudes have been normalized by the maximum DF power in each row. Frequency whitening has not been applied to the data, though it can be verified that the directionality does not change noticeably; only the weaker sources are amplified.

bands is clearly different for the entire data set and the subset, and for the latter, it lies at the northwest direction in both bands. In the GP band, however, the maximum power comes from a different direction. This may seem inconsistent, but it can be explained by the fact that the beamforming was applied to the entire 10 min time series (i.e., not for the frequency bands separately), giving a maximum that still lies at the northwest.

Table 1. Station Names of the BN Array (Figure 1), Associated Trace Numbers in the PSF and Virtual Source Function (Figures 9 and 10), and the Required Interpolation per Month

\begin{tabular}{lcc} 
Station Name & Trace in PSF & Interpolation \\
\hline BN01 & 1 & - \\
BN02 & 2 & July, August, and September \\
BN04 & 3 & - \\
BN05 & 4 & May, July, August, and September \\
BN06 & 5 & - \\
BN07 & 6 & May, July, August, and September \\
BN08 & 7 & - \\
BN09 & 8 & - \\
BN10 & 9 & May \\
BN11 & 10 & July, August, and September \\
BN12 & 11 & - \\
BN13 & 12 & - \\
BN13A & 13 & - \\
BN14 & 14 & - \\
BN15 & 15 & - \\
BN16 & 16 & - \\
BN17 & 17 & - \\
BN18 & 18 & - \\
BN19 & 19 & - \\
BN20 & 20 & - \\
BN21 & 21 & - \\
BN22 & 22 & - \\
BN23 & 23 & - \\
\hline
\end{tabular}


Table 2. Station Names of the BS Array (Figure 1), Associated Trace Numbers in the Virtual Shot Seismograms (Figures 5, 6, and 15), and Stations That Were Left Out Since They Were Partially or Fully Offline

\begin{tabular}{lcc} 
Station Name & Trace in Seismograms & Incorporated \\
\hline BS01 & 1 & $\mathrm{x}$ \\
BS02 & 2 & $\mathrm{x}$ \\
BS03 & 3 & $\mathrm{x}$ \\
BS04 & 4 & - \\
BS04M & 5 & $\mathrm{x}$ \\
BS05 & 6 & $\mathrm{x}$ \\
BS06 & 7 & $\mathrm{x}$ \\
BS07 & 8 & $\mathrm{x}$ \\
BS09 & 9 & $\mathrm{x}$ \\
BS10 & 10 & $\mathrm{x}$ \\
BS11 & 11 & $\mathrm{x}$ \\
BS12 & 12 & - \\
BS12M & 13 & $\mathrm{x}$ \\
BS13 & 14 & $\mathrm{x}$ \\
BS14 & 15 & $\mathrm{x}$ \\
BS15 & 16 & $\mathrm{x}$ \\
BS15A & 17 & $\mathrm{x}$ \\
BS16 & 18 & $\mathrm{x}$ \\
BS17 & 19 & $\mathrm{x}$ \\
BS18 & 20 & $\mathrm{x}$ \\
BS19 & 21 & $\mathrm{x}$ \\
BS21 & 22 & - \\
BS22 & 23 & $\mathrm{x}$ \\
\hline
\end{tabular}

The selected data subset mainly contains inward propagating Rayleigh waves. In total, we used 411 time windows having noise from the proper direction, which is $2.5 \%$ of a complete 4 month data set (May, July, August, and September 2006; 16,704 time windows). We used these 4 months because the number of appropriate time windows was relatively high, the SNR in the cross correlations was good, the number of stations online over the entire period was high and relatively constant, and to avoid adjacent BN stations being offline. The latter would raise problems in the interpolation to fill in empty traces in the PSF (discussed below).

To build the PSF (equation (10)), we cross correlated the data subset of all stations in the BN array with that of all other BN stations. We also cross correlated the data subset of each BN station with that of all BS stations to build the CCF (equation (9)). It should be emphasized that all stations need to have the same noise input to make the functions consistent for MDD (which follows from equation (6)). In Tables 1 and 2, we show

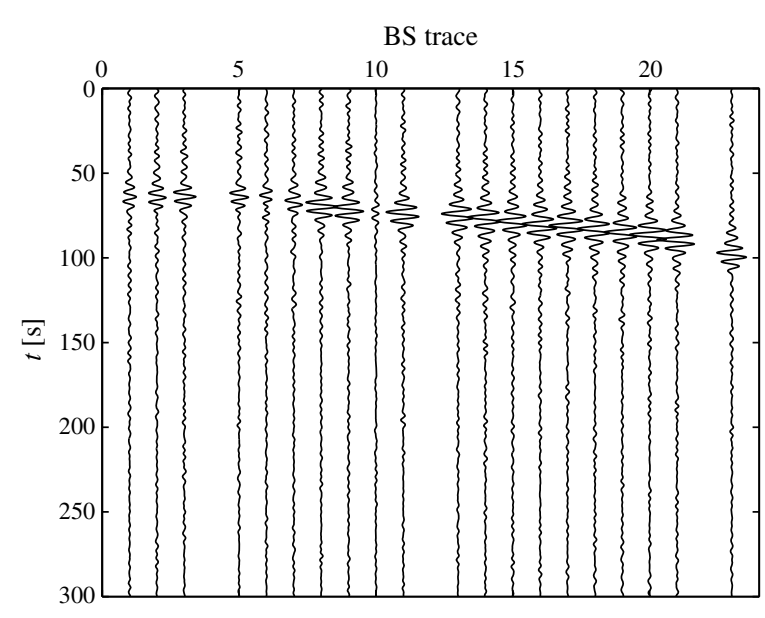

Figure 5. Virtual shot seismogram for receivers along the BS array obtained by MDD. The virtual source is at station BN06. Note that the source-receiver distance does not increase linearly. which stations were online during the entire period. The data of station BN07 were removed because of poor quality. We interpolated the empty traces in the PSF on a month basis using the traces of adjacent stations before stacking the different months. Data related to BS stations offline over part of the 4 month period were simply left out of the CCF (see Table 2 for details), giving empty traces for these stations in the virtual shot seismograms (Figures 5, 6 , and 15).

We note that, by cutting the continuous noise data into $10 \mathrm{~min}$ time windows and by choosing windows with a dominant source (to the northwest), the assumption of simultaneously acting sources as described in section 2 might seem overstated. The 


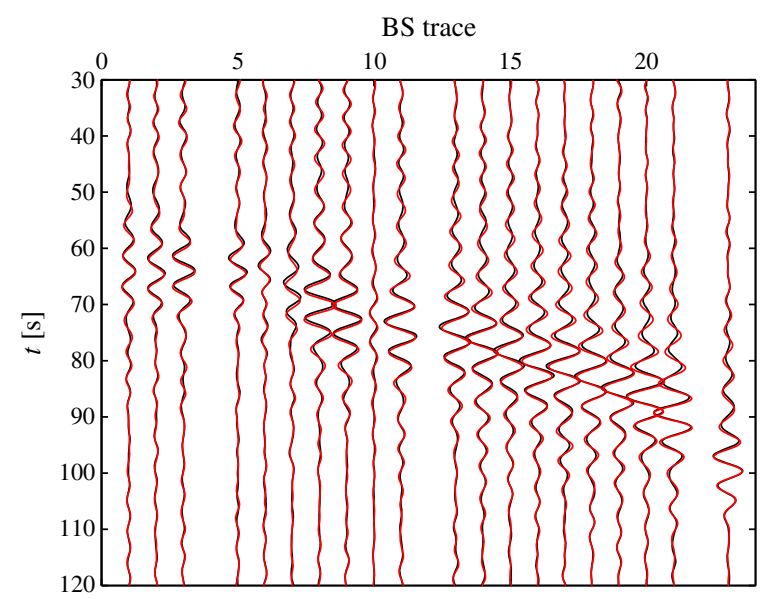

Figure 6. Comparison of MDD (black) and CC1 results (red) for receivers along the $\mathrm{BS}$ array and the virtual source at station BN06 (see Table 3 for explanation of methods). Note that the source-receiver distance does not increase linearly. assumption of sequential sources might be appropriate as well (cf. equations (13) and (14)), but it can still be true that multiple sources (all lying to the northwest of the BN array) are active within a selected time window. We probably have a mixed case where some of the time windows have a single source while others have multiple sources.

\section{Results of MDD on Batholiths Data}

In this section, we show the result of MDD applied to the data selected using beamforming (MDD result). In section 4.1, we compare it to the results obtained using the CC method applied to the same data subset, both without and with frequency whitening [Bensen et al., 2007] applied to

the noise prior to cross correlation (CC1 and CC2 results, respectively). We first compare the MDD and CC1 results to illustrate the improvement achieved by the MDD operation. Then, we compare the MDD and CC2 results to illustrate the obtained quality against that of the CC method with the conventional processing technique applied (i.e., frequency whitening, in addition to the running-absolute-mean normalization over time shown in equation (18)). For completeness, we compare the MDD result with that obtained using the CC method applied to all data recorded during the 4 months (CC3 result, which includes spectral whitening as well); see section 4.2. We also present dispersion curves extracted from the different results to illustrate the performance of the different methods. The station names corresponding to the trace numbers referred to in the figures and the text are given in Tables 1 and 2, and the methods discussed in this section are specified in Table 3.

\subsection{MDD Versus CC1 and CC2}

In Figure 5, a virtual shot seismogram obtained using MDD (equation (16)) is shown. The associated virtual source is at station BN06 (cf. Table 1 and Figure 1). For this station, the MDD result has the highest quality: well-developed waveforms with relatively constant amplitudes, especially at the eastern part of the BS array. This is due to the fact that the associated direction of propagation $\left[\operatorname{in} \bar{G}_{\mathrm{d}}\left(\mathbf{x}_{R}, \mathbf{x}, t\right)\right.$ ] is aligned with the dominant direction of the noise, which comes from the coastline (cf. Figures 1 and 4).

A comparison with the CC1 result is shown in Figure 6, where we zoomed on the surface waveforms. The differences between the MDD and CC1 results are not extreme, but the MDD waveforms are better localized (more focused in time). This feature is more pronounced in Figure 7, which contains a comparison of the 21st BS traces, both having well-developed Rayleigh wave arrivals. In Figure 7, the SNR of the MDD result looks slightly higher, and this is confirmed by the quantitative comparison in Figure 8 (the lines associated with other methods are referred to below); here the definition of SNR as proposed by Kao et al. [2013] has

Table 3. Different Methods Discussed in Section 4, Including the Details (Specific Data and Additional Operations); CC Refers to Cross Correlation, MDD to Multidimensional Deconvolution, and TD to Temporal Deconvolution ${ }^{\mathrm{a}}$

\begin{tabular}{lr} 
Method & Details \\
\hline CC1 & CC applied to data subset, without frequency whitening of the noise \\
MDD & MDD applied to CC1 result \\
TD & TD applied to CC1 result \\
CC2 & CC applied to data subset, with frequency whitening of the noise \\
CC3 & CC applied to entire data set, with frequency whitening of the noise \\
\hline
\end{tabular}

${ }^{a}$ In all methods, the data were normalized according to equation (18). 


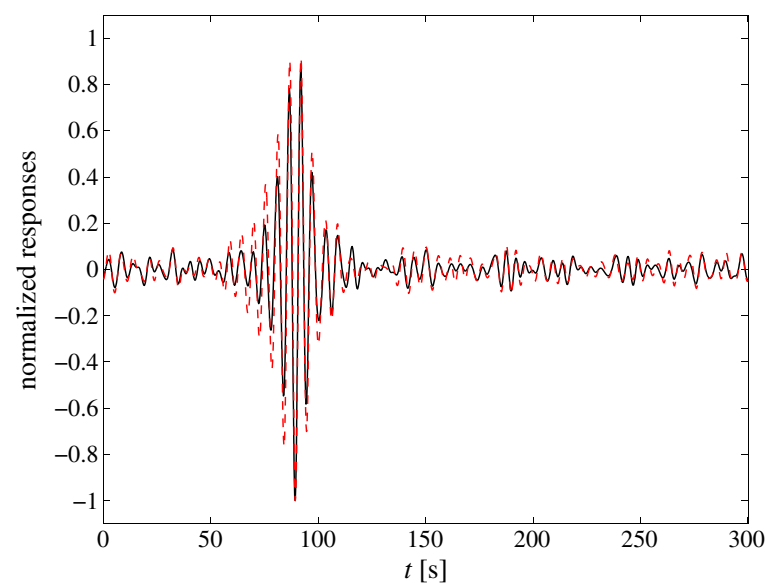

Figure 7. Comparison of MDD (black) and CC1 (red, dashed) results (21st BS traces) for the virtual source at station BN06. been adopted, which states that the SNR is the ratio between the largest absolute amplitude and the mean absolute amplitude. Clearly, the SNR of the MDD result is significantly higher than that of the CC1 result for all BS stations (in this virtual shot seismogram). With regard to Figure 8 , we note that the increase in SNR with increasing BS trace number corresponds with our statement above about the well-developed waveforms at the eastern part of the BS array due to the directionality of the noise. We also note that MDD applies phase corrections to the smaller offset responses (i.e., time shifts in the observations at the west part of the BS array; Figure 6).

The PSF used in the deconvolution is shown in Figure 9, with $\mathbf{x}^{\prime}$ kept fixed at the virtual source BN06 (cf. equation (10)). The function shows a spatial and temporal smearing of the virtual source, which can be caused by a nonuniform noise source distribution, correlated noise sources, and/or losses, making the CC result less accurate (see also section 2). The virtual source function $\Upsilon$ shown in Figure 10 exhibits a much better focus (cf. equation (17) and explanation thereafter), particularly in space; the frequency spectrum of the PSF (not shown) is relatively flat, giving minor improvement in temporal focusing. The better focus indicates that the virtual source response obtained using MDD is more accurate. Here we note that the improvement has been reached even though interpolation in the PSF was required to fill in data gaps related to the stations adjacent to the virtual source (BN05 and BN07; see also section 3).

As indicated, the waveforms obtained using MDD are more focused in time (see Figures 6 and 7). This is not only due to the temporal deconvolution (TD) of the source signature (cf. equations (4) and (16)), as demonstrated by the comparison with the MDD result in Figure 11. The TD result in Figure 11 was obtained by deconvolving the CCF by a one-dimensional PSF computed using the observation at the virtual source only (i.e., $\mathbf{x}=\mathbf{x}^{\prime}=$ constant in equation (10)). Clearly, the spatial deconvolution further improves the obtained virtual source response. Furthermore, note that the SNR of the MDD result is significantly higher than that of the TD result (see Figure 8).

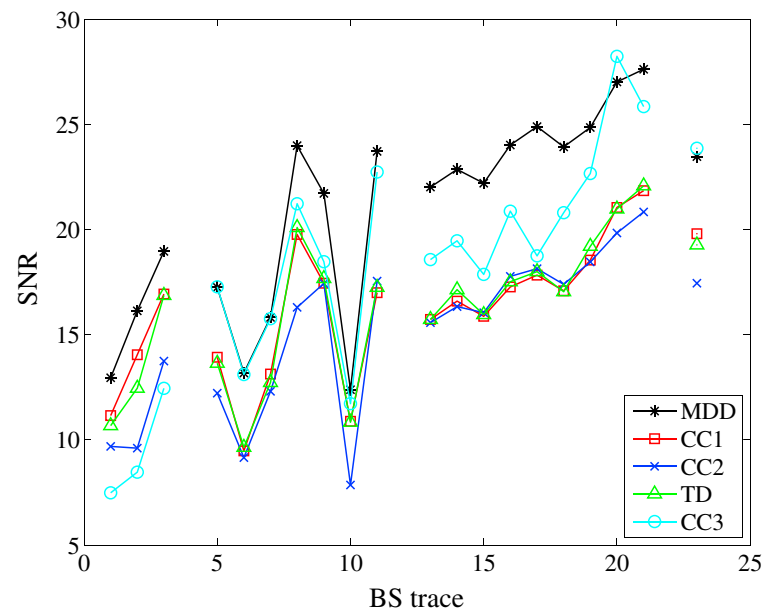

Figure 8. Signal-to-noise ratio (SNR) for receivers along the BS array and the virtual source at station BN06; different lines correspond to the different methods indicated in the legend (see also Table 3). The gaps correspond to BS stations without data (see also Figure 5 and Table 2).
The effect of the spatial deconvolution is most pronounced in the frequency-wave number $(f, k)$ domain panels of the responses (Figure 12), which were obtained using the Fourier transform over time and space (i.e., the BS array); we note that the spatial transform proposed by Park et al. [1998] was used (i.e., a discretized spatial transform applied to the amplitude-normalized space-frequency domain responses) and that the medium is implicitly assumed invariant in horizontal direction when it is applied. MDD fills in the spectral gap/notch between the SF and DF microseism bands visible in the $\mathrm{CC} 1$ result. Although this gap is not equally clear for virtual shot seismograms related to adjacent BN stations (not shown), it is likely that it occurs due to an inappropriate dominant direction of illumination (see Figure 4, 


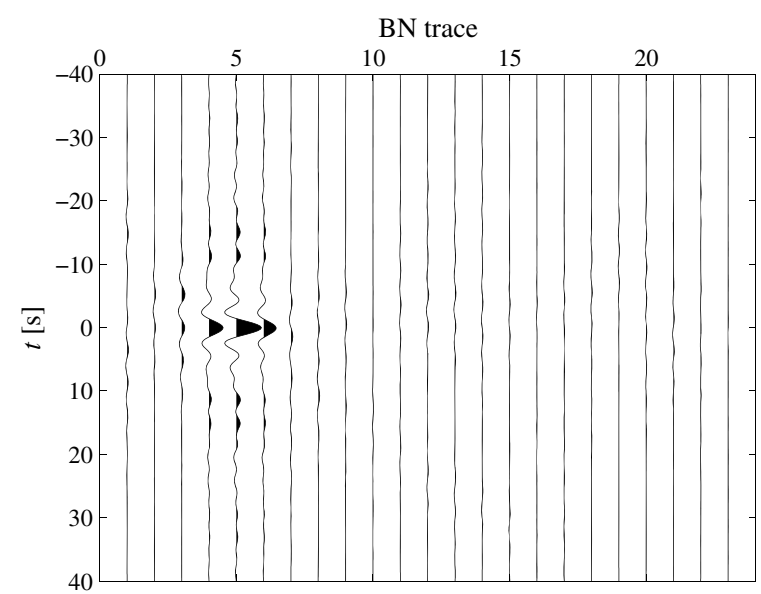

Figure 9. Spatial and temporal distribution of the PSF $\Gamma\left(\mathbf{x}, \mathbf{x}^{\prime}, t\right)$, where $\mathbf{x}^{\prime}$ is fixed at the virtual source (station BN06) and $\mathbf{x}$ varies along the $\mathrm{BN}$ array.
GP band). The TD does not fill in the missing wave numbers; it only improves the CC1 result slightly compared to MDD.

It should be noted that frequency whitening of the noise subset selected using beamforming (i.e., CC2 result), as conventionally applied in the CC method prior to cross correlation, does improve the CC1 result by amplifying the weaker sources (we verified that the directionality itself as shown in Figure 4 is not changed noticeably by the frequency whitening). The spectral gap can be filled without applying MDD; the $f, k$ domain panel (not incorporated in Figure 12 for brevity) shows a similar pattern as that of the CC3 response, which is referred to in section 4.2. However, the phase differences compared to the MDD result remain (not shown, but similar as in Figure 6), and the SNR of the CC2 result is even slightly lower than that of the CC1 result for most stations (Figure 8), which illustrates that MDD is not a trivial processing step (the SNR of the MDD result is significantly higher than that of the CC1 and CC2 results).

To further support the latter statement, we compare the phase velocities (i.e., related to a horizontally invariant medium) picked from the peaks in the $f, k$ domain panels. We leave out the CC1 result, as it gives quite poor results in the spectral gap, and incorporate the CC2 result instead. Figure 13 shows average phase velocities and estimates of the spread (i.e., twice the standard deviation) obtained from responses with virtual sources at stations BN01, BN04, and BN06; we did not include stations BN02, BN05, and BN07 to guarantee the same amount of data in the comparison (see Table 1), and we left out stations beyond BN07 due to the bad quality of the virtual source responses, which is due to the specific dominant direction of the noise (as previously mentioned). The phase velocities obtained from the MDD result are obviously closer to the regional model [Kao et al., 2013] than those of the CC2 result; moreover, the spread is smaller, and the jump around $0.2 \mathrm{~Hz}$ (discussed in more detail below) is absent. We note that these findings do not necessarily imply that the phase velocities obtained using MDD are more accurate because the model itself originates from an inversion procedure which introduces uncertainties, but the findings are consistent with those obtained using numerical experiments where the true phase velocities were known

[Wapenaar et al., 2011a]. The phase velocities extracted from the TD result,

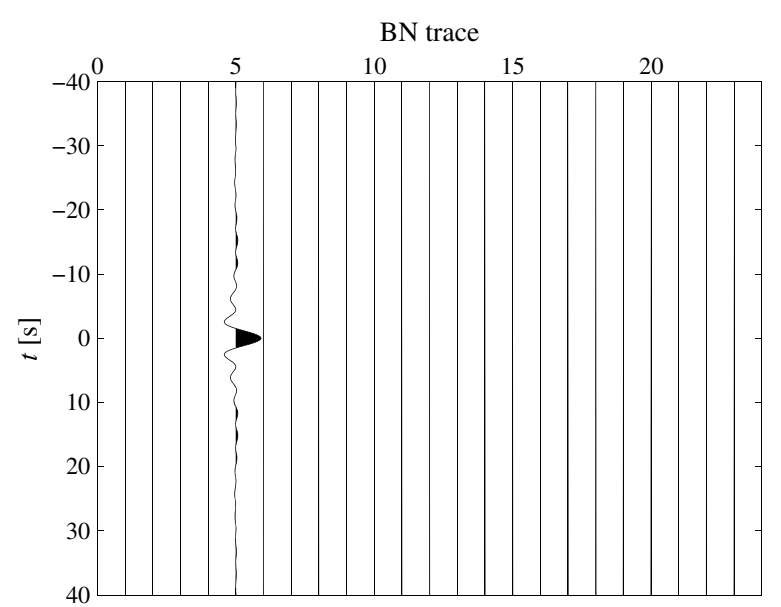

Figure 10. Spatial and temporal distribution of the virtual source function $\Upsilon\left(\mathbf{x}, \mathbf{x}^{\prime}, t\right)$, where $\mathbf{x}^{\prime}$ is fixed at the virtual source (station $\mathrm{BN} 06)$ and $\mathbf{x}$ varies along the BN array. which are also included in Figure 13, have similar deviation from the regional model as those of the CC2 result, but the spread is slightly larger; as virtual shots other than BN06 (i.e., BN01 and BN04) have been incorporated to compute the average, the phase velocities are quite close to the regional model for frequencies in the spectral gap.

The jump in the average phase velocities obtained from the CC2 result (Figure 13) originates from another virtual source than BN06. This is clear from Figure 14, which compares the $f, k$ domain panels (CC2) for virtual sources at stations BN01 and BN06. A possible cause of the jump is that, for a narrow frequency band, the illumination 


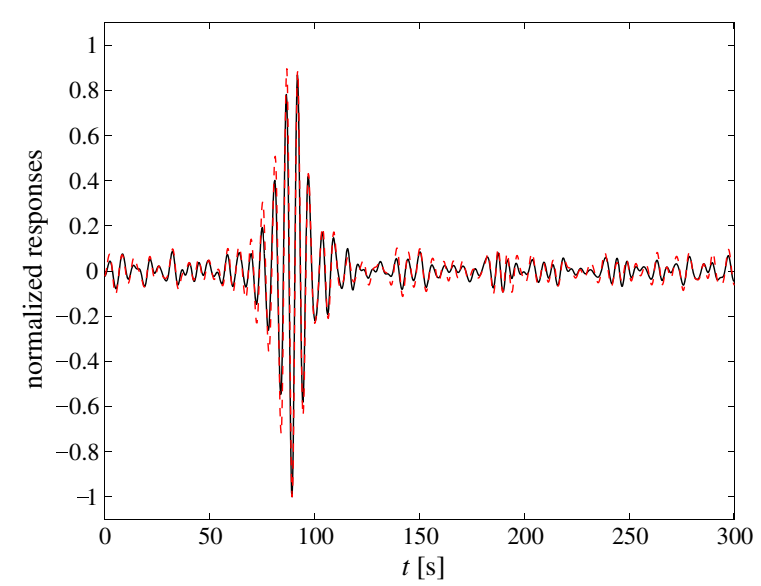

Figure 11. Comparison of MDD (black) and TD (red, dashed) results (21st BS traces) for the virtual source at station BN06. is biased to a source area outside the stationary-phase region. This leads to a reconstruction with too small travel times and, as a result, overestimated phase velocities. In principle, it could also be a jump to a higher-mode surface wave or occur due to a lateral heterogeneity, which gives a shorter propagation path for a specific frequency; however, this is an unlikely scenario as the MDD result does not exhibit this jump (as confirmed by Figure 13).

To conclude, by applying MDD to the CC1 result, one obtains a virtual source response with relatively high SNR and corrected for irregular illumination. Conclusive statements about the accuracy of the extracted phase velocities compared to those of the CC1 and CC2 results cannot be made, as the regional model is subject to uncertainties.

\subsection{MDD Versus CC3}

The time domain CC result that includes all noise recorded in the 4 months (CC3 result) is shown in Figures 15 and 16, together with the MDD result. Note that, for the CC3 result, the conventional frequency whitening was applied to the noise prior to cross correlation; we also checked 1 bit normalization and obtained a very similar CC3 result, which is confirmed by Bensen et al. [2007].

The CC3 waveforms are better localized, which is due to the broader bandwidth of the noise. The beamforming selects the noise from a limited aperture and includes a cluster of microseism sources with one specific peak frequency. In the CC3 result, many sources with different peak frequencies are present due to varying coastal/continental shelf morphology, thus giving a more broadband virtual source response. A comparison of the amplitude spectra of the 21 st BS traces is given in Figure 17. Furthermore,
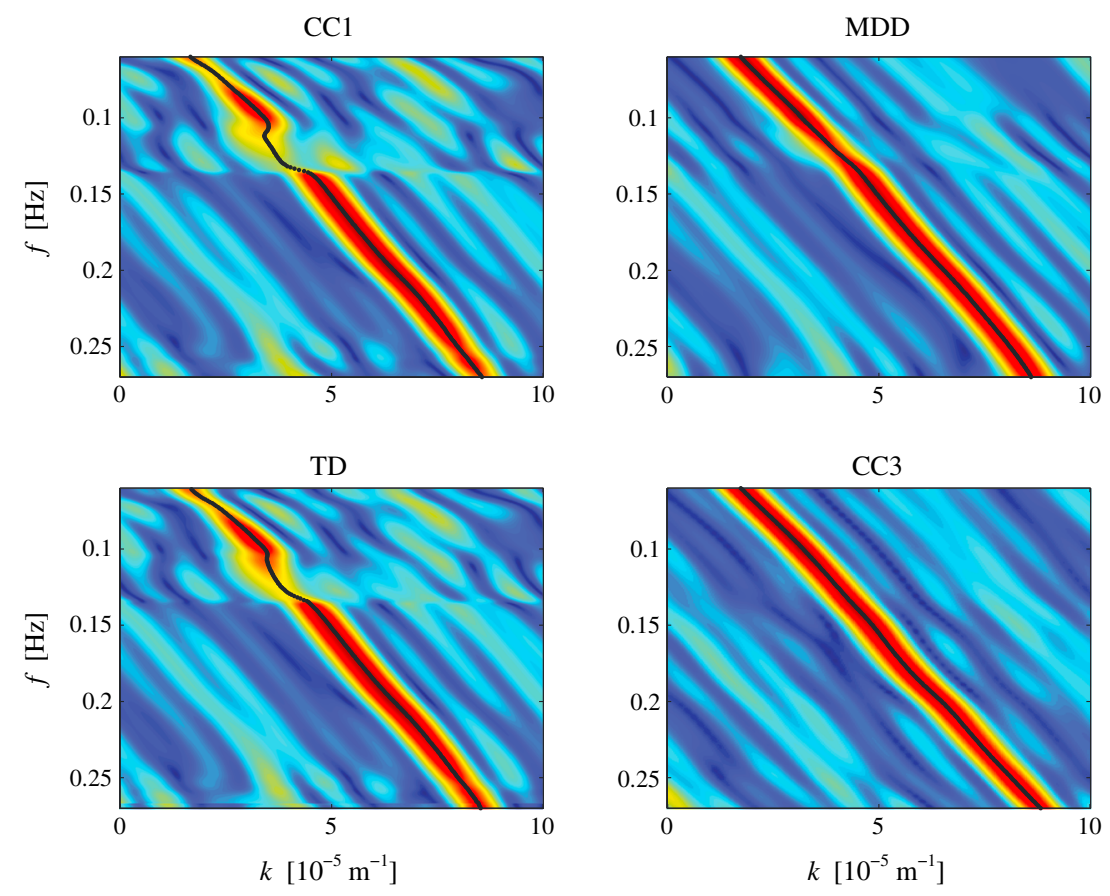

Figure 12. Frequency-wave number $(f, k)$ domain panels of the CC1, MDD, TD, and CC3 results (see Table 3 ) for the virtual source at station BN06. Black dots indicate the picked phase-velocity values. 

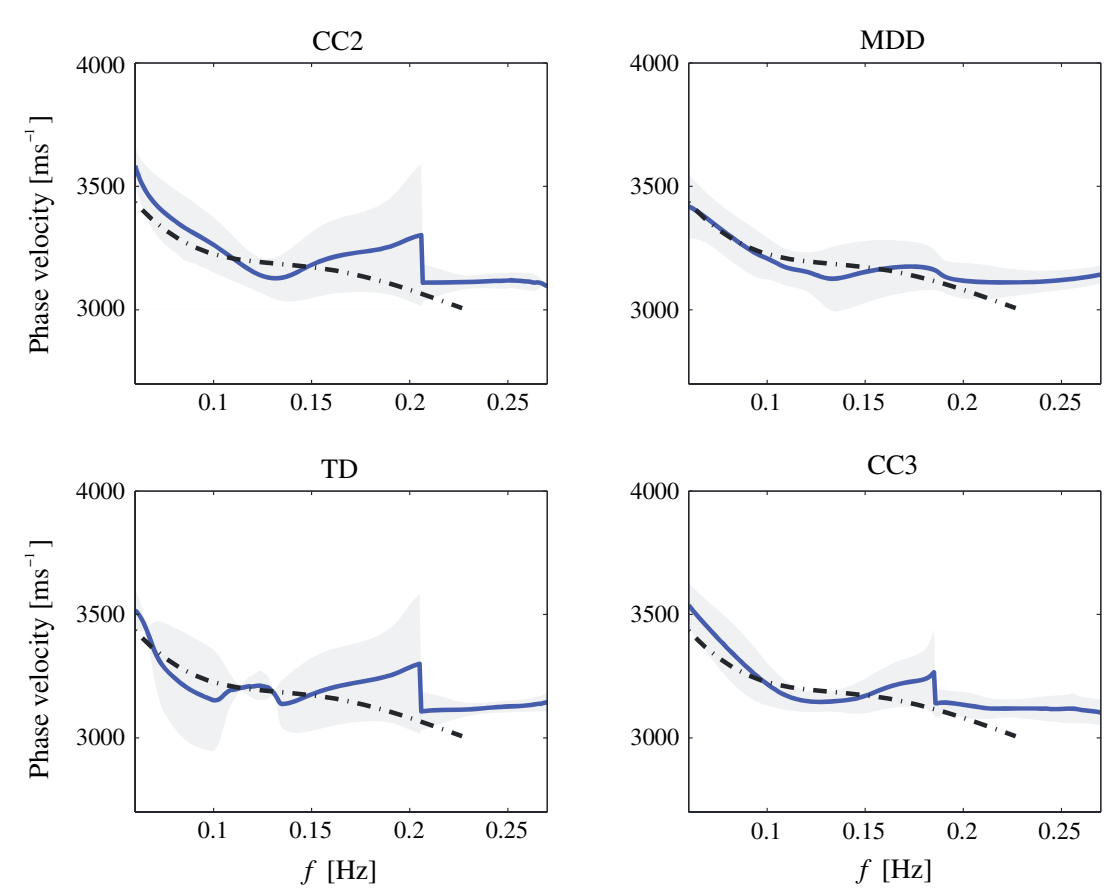

Figure 13. Average phase velocities (solid blue lines) based on virtual sources at stations BN01, BN04, and BN06 and the associated spreads (i.e., twice the standard deviation indicated by the shaded areas) estimated from the CC2, MDD, TD, and CC3 results (see Table 3). The phase velocity according to the corresponding regional model [Kao et al., 2013] has been included for comparison (dash-dotted line).

the CC3 waveforms show stronger variations in amplitude across the BS array compared to the MDD waveforms (Figure 15). This is likely due to the nonisotropic directionality of the noise over the entire period (cf. panels in Figure 4 (top) and Figure 4 (bottom); note that the frequency whitening does not change the directionality), which does not affect the MDD result, as the noise from noncoherent directions was left out to a large extent. Note that conclusions about amplitude corrections by MDD cannot be drawn here, as different data were used for MDD and CC3. In addition, the CC3 result contains artifacts at early times, which is probably also due to the directionality of the noise. These artifacts are the main cause of the poorer SNR of the $\mathrm{CC} 3$ result compared to that of the MDD result (see Figure 8). Overall though, the CC3 result has better SNR than the CC results based on the data subset (CC1 and CC2).

Finally, it is clear from Figure 12 that using all noise is another way to fill in the spectral gap in the $f, k$ domain panel, though this was also achieved in the CC2 result, as mentioned in section 4.1. The frequency whitening plays a major role here, rather than the amount of data. While the average phase velocities extracted from the $\mathrm{CC} 3$ result are closer to the regional model than those obtained from the $\mathrm{CC} 2$ result, the spread is also lower (Figure 13), which demonstrates the advantage of using more data. The phase velocities based on the
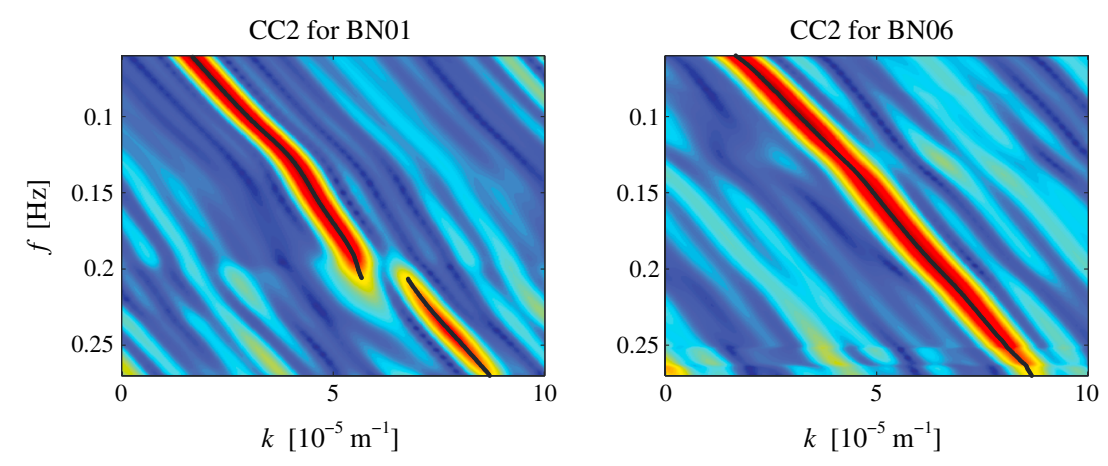

Figure 14. Frequency-wave number $(f, k)$ domain panels of the $C C 2$ results (see Table 3 ) for the virtual sources at stations BN01 and BN06. Black dots indicate the picked phase-velocity values. 


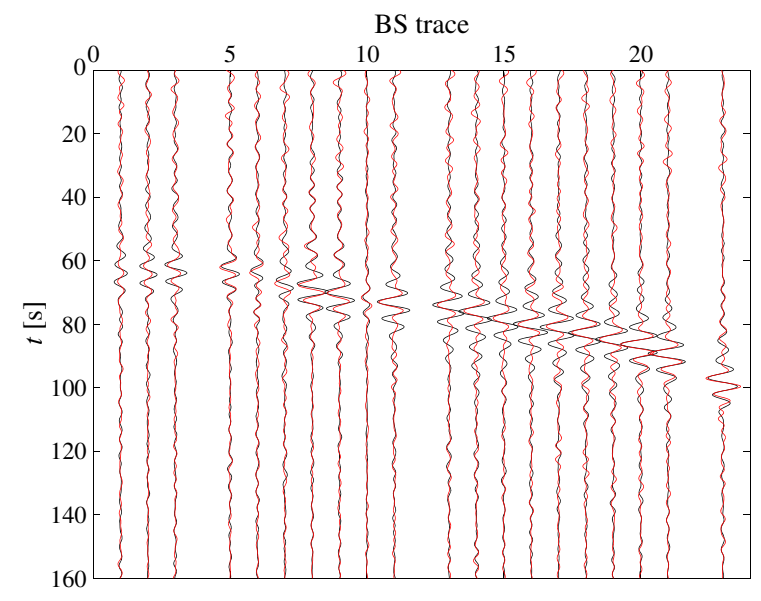

Figure 15. Comparison of MDD (black) and CC3 results (red), with receivers along the BS array and the virtual source at station BN06. Note that the source-receiver distance does not increase linearly.
MDD result are even slightly more close to the regional model than those from the CC3 result (particularly for the lower frequencies), while the spread is slightly smaller for some frequencies (especially around the phase-velocity jump, which is still present for $\mathrm{CC} 3$, though it has been suppressed a little compared to (C2) and slightly larger for others. However, MDD reaches this using only a small carefully selected subset of the entire data set, which shows the strength of the MDD method.

\section{Discussion}

We now discuss the influence of the sampling properties of the BN array on the MDD result, the possibility to extract the surface wave attenuation factor from the obtained virtual source responses and the ability of the MDD method to cope with mode conversions due to scattering.

Regarding the sampling properties, it is obvious that the spacing of the BN stations should be such that the integral in equation (8) can be approximated with small error. This implies that the largest wave number present in the PSF should not exceed the Nyquist wave number $k_{N}=1 /(2 \Delta)$, where $\Delta$ denotes the spatial sampling interval. It can be verified, however, that the PSF as shown in Figure 9 is slightly aliased with respect to the Nyquist wave number calculated based on the average station spacing in the BN array. This makes the phase velocities extracted from the MDD responses somewhat unreliable for the highest frequencies. Apart from a slightly increasing average value, no strange effects are observed in Figure 13, and we therefore conclude that the influence of the aliasing is minor. Furthermore, the fact that the array has finite extent also influences the quality of the MDD result, particularly because the chosen virtual source (station BN06) lies rather close to one of the edges (the virtual sources incorporated in the dispersion analysis are even closer; see section 4). Future arrays designed specifically for MDD should be longer, so that truncation effects can be minimized.

Regarding the surface wave attenuation factor, it is well-known that it is challenging to estimate its magnitude from interferometric Green's function estimates. Nonuniform noise source distributions lead to a bias in the estimates of the attenuation when extracted from CC results [Lawrence et al., 2006; Tsai, 2009;

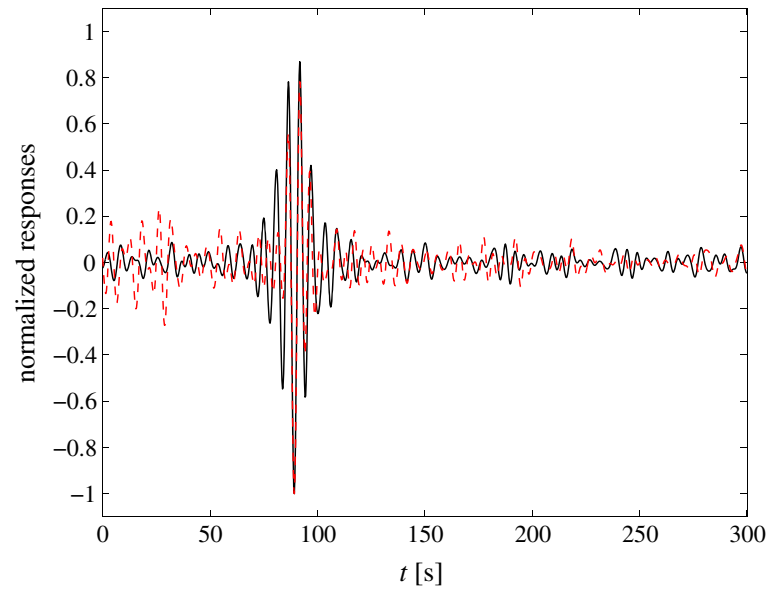

Figure 16. Comparison of MDD (black) and CC3 (red, dashed) results (21st BS traces) for the virtual source at station BN06. Cupillard and Capdeville, 2010]. In media with severe scattering, the so-called $C^{3}$ method [Stehly et al., 2008] effectively reduces bias and allows for more reliable attenuation estimates from noise [Zhang and Yang, 2013], which is likely because the coda of noise cross correlation contains more diffused noise energy. With the correct network configuration, the $C^{3}$ processing effectively makes the noise source distribution more uniform. Another way to assess the attenuation factor is to use the noise coherency function [Prieto et al., 2009; Lawrence and Prieto, 2011; Weemstra et al., 2013]. However, the accuracy of the results also depend on the distribution of noise sources, so that knowledge of this distribution is required [Tsai, 2011]. As an 


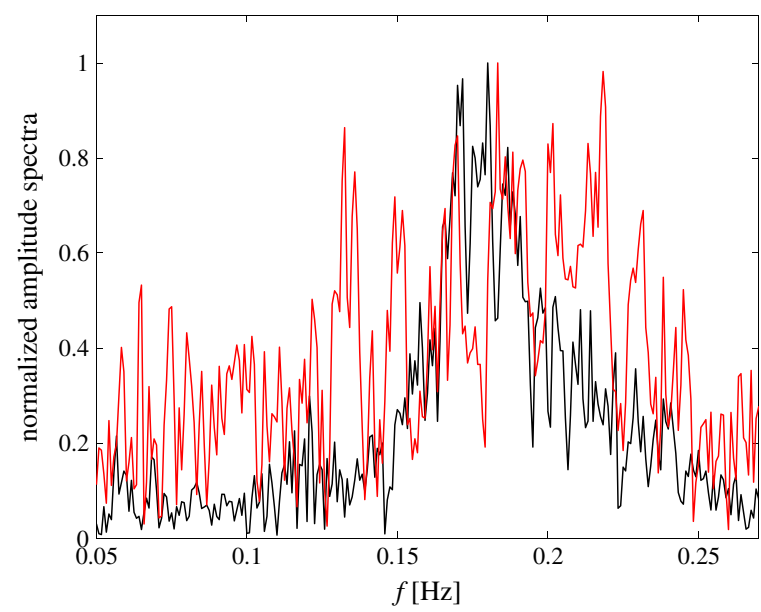

Figure 17. Spectra of the MDD (black) and CC3 (red) virtual source responses (21st BS traces), for the virtual source at station BN06. alternative, MDD could be very useful to estimate the attenuation factor. Losses are incorporated in a natural way, and the effect of irregularities in the source distribution can be compensated for (see also sections 1 and 2).

In order to estimate the attenuation factor from the MDD result using methods such as the spectral ratio [Jeng et al., 1999], the data processing must handle the amplitude information in an appropriate way.

Regarding the normalization of the raw data, the response to each source should ideally be normalized to one so that the cross correlation of high-amplitude noise does not overwhelm that related to more quiet conditions; per source, the data of all stations must be scaled using a single global value. Such normalization is, however, practically impossible with multiple sources of different source strengths being active in the same 10 min time window. Using only one global normalization value per time window would normalize the associated dominating source. This may work as long as there are many windows so that treating only the dominating sources yields sufficient illumination. However, from our tests it appeared that applying such global normalization has the drawback that stations with local amplification issues become useless. For some stations, the obtained noise cross correlation had very low SNR, which is likely due to locally generated noise. In particular, we had to remove the data from a BN station adjacent to stations that were offline, implying major difficulties for the interpolation in the PSF (see section 3). In order to get traces of sufficient quality in the PSF, we had to apply the local normalization (i.e., per station) described in section 3, meaning that every sample in the raw data is scaled using the amplitudes of the surrounding time samples (see equation (18)). By applying this type of normalization, we could down weight the local noise. Clearly, this makes the current MDD result inappropriate for assessing the surface wave attenuation factor. Future work should therefore focus on the attenuation issue.

Strictly speaking, the MDD scheme for scalar waves discussed in this paper only works for single-mode surface waves and cannot handle mode conversions inside the domain $D_{m}$ (Figure 2). However, scattering inside $D_{m}$ can be handled [Wapenaar et al., 2011b], as long as the mode conversions are small. Mode conversions outside $D_{m}$ are allowed provided that the inward propagating field at boundary $S_{m}$ is dominated by single-mode surface waves, which is often the case. In the case of an inward propagating field containing multiple surface wave modes, the MDD scheme for vectorial fields could be applied [van Dalen et al., 2014].

\section{Conclusions}

This paper presents the results of seismic interferometry by multidimensional deconvolution (MDD) applied to ambient noise surface wave observations. The data were taken from the Batholiths experiment [Calkins et al., 2010], which was a passive deployment of broadband seismic sensors in British Columbia (Canada; 2005-2006), with noise primarily of oceanic origin. The deployment consisted of two approximately linear station lines, the north and south arrays. Using 4 months of recordings-bandpass filtered between 0.05 and $0.27 \mathrm{~Hz}$ - fundamental-mode Rayleigh waves could be retrieved using cross correlations. For the application of MDD, we selected the noise time windows dominated by waves that traversed the northern array before reaching the southern using beamforming, which is required to build the point-spread function (PSF); we thus selected a subset containing $2.5 \%$ of all data. The estimated virtual source response by MDD was then obtained by deconvolving the cross correlation result associated with this subset (CC1 result; see Table 3) by the PSF.

We found that the MDD waveforms are more localized in time and have significantly better SNR compared to the CC1 result. In addition, MDD applies phase corrections in the virtual shot seismogram. We also found 
that MDD fills in a spectral gap in the $f, k$ domain between the single-frequency and double-frequency microseism bands, which is primarily the result of the spatial deconvolution. Frequency whitening of the selected noise data also fills in the spectral gap (CC2 result; see Table 3), but the phase differences compared to the MDD result remain and the SNR remains lower. In addition, the average phase velocities obtained from the MDD result are closer to the regional model than those of the CC2 result, plus the spread across different virtual sources is smaller.

Compared to the result obtained using the cross correlation of all data (CC3 result; see Table 3), which we included for comparison, the SNR of the MDD result is higher as well. This is due to the nonisotropic directionality of the noise, leading to amplitude variations over the virtual shot seismograms and artifacts at early times. The average phase velocities based on the MDD result are still closer to the regional model than those of the CC3 result (particularly for the lower frequencies), while the spread is comparable; this does not necessarily imply higher accuracy due to applying MDD, but the findings are consistent with those obtained using numerical experiments where the true phase velocities were known [Wapenaar et al., 2011a]. MDD reaches these phase-velocity estimates using only a small subset (2.5\%) of the entire data set. Although the subset was carefully selected, this illustrates the strength of the MDD method.

We were not able to estimate the surface wave attenuation factor from the current MDD result. The reason is that the required processing for the data set involved normalization of the raw data for each station individually, implying that the amplitude information is lost to some extent. In theory, however, losses are properly captured in the MDD scheme, and it therefore has the potential to provide realistic attenuation factors.

\section{Acknowledgments}

K.N.v.D. and E.N.R. were sponsored by The Netherlands Research Centre for Integrated Solid Earth Sciences (ISES). T.D.M. was financially supported by ERC advanced grant 226837 and the National Science Foundation under award 1144883. The facilities of the Incorporated Research Institutions for Seismology (IRIS), in particular the IRIS Data Management Center (DMC; http://www.iris.edu/dms/nodes/dmc/), were used for accessing the waveforms and metadata required in this study.

\section{References}

Berkhout, A. J. (1982), Seismic Migration: Imaging of Acoustic Energy by Wave Field Extrapolation, Elsevier, Amsterdam, Netherlands. Bensen, G. D., M. H. Ritzwoller, M. P. Barmin, A. L. Levshin, F. Lin, M. P. Moschetti, N. M. Shapiro, and Y. Yang (2007), Processing seismic ambient noise data to obtain reliable broad-band surface wave dispersion measurements, Geophys. J. Int., 169, 1239-1260.

Bromirski, P. D., R. A. Stephen, and P. Gerstoft (2013), Are deep-ocean-generated surface-wave microseisms observed on land?, J. Geophys. Res. Solid Earth, 118, 3610-3629, doi:10.1002/jgrb.50268.

Calkins, J. A., G. Zandt, J. Girardi, K. Dueker, G. E. Gehrels, and M. N. Ducea (2010), Characterization of the crust of the Coast Mountains Batholith, British Columbia, from P to S converted seismic waves and petrologic modeling, Earth Planet. Sci. Lett., 289, 145-155.

Campillo, M., and A. Paul (2003), Long-range correlations in the diffuse seismic coda, Science, 299, 547-549.

Cupillard, P., and Y. Capdeville (2010), On the amplitude of surface waves obtained by noise correlation and the capability to recover the attenuation: A numerical approach, Geophys. J. Int., 181, 1687-1700.

Gallot, T., S. Catheline, P. Roux, and M. Campillo (2012), A passive inverse filter for Green's function retrieval, J. Acoust. Soc. Am., 131, EL21-EL27.

Gerstoft, P., K. G. Sabra, P. Roux, W. A. Kuperman, and M. C. Fehler (2006), Green's functions extraction and surface-wave tomography from microseisms in southern California, Geophysics, 71, SI23-SI31.

Halliday, D., and A. Curtis (2008), Seismic interferometry, surface waves and source distribution, Geophys. J. Int., 175, $1067-1087$.

Jeng, Y., J.-Y. Tsai, and S.-H. Chen (1999), An improved method of determining near-surface Q, Geophysics, 64, $1608-1617$.

Kao, H., Y. Behr, C. A. Currie, R. Hyndman, J. Townend, F.-C. Lin, M. H. Ritzwoller, S.-J. Shan, and J. He (2013), Ambient seismic noise tomography of Canada and adjacent regions: Part I. Crustal structures, J. Geophys. Res. Solid Earth, 118, 5865-5887, doi:10.1002/2013JB010535.

Lacoss, R., E. Kelly, and M. Toksoz (1969), Estimation of seismic noise structure using arrays, Geophysics, 34, 21-38.

Larose, E., L. Margerin, A. Derode, B. van Tiggelen, M. Campillo, N. Shapiro, A. Paul, L. Stehly, and M. Tanter (2006), Correlation of random wavefields: An interdisciplinary review, Geophysics, 71, SI11-SI21.

Lawrence, J. F., and G. A. Prieto (2011), Attenuation tomography of the western United States from ambient seismic noise, J. Geophys. Res., 116, B06302, doi:10.1029/2010JB007836.

Lawrence, J. F., P. M. Shearer, and G. Masters (2006), Mapping attenuation beneath North America using waveform cross-correlation and cluster analysis, Geophys. Res. Lett., 33, L07315, doi:10.1029/2006GL025813.

Mulargia, F. (2012), The seismic noise wavefield is not diffuse, J. Acoust. Soc. Am., 131, 2853-2858.

Park, C. B., R. D. Miller, and J. Xia (1998), Imaging dispersion curves of surface waves on multi-channel record, SEG Expanded Abstracts, pp. 1377-1380.

Prieto, G. A., J. F. Lawrence, and G. C. Beroza (2009), Anelastic Earth structure from the coherency of the ambient seismic field, J. Geophys. Res., 114, B07303, doi:10.1029/2008JB006067.

Rost, S., and C. Thomas (2002), Array seismology: Methods and applications, Rev. Geophys., 40, 1008, doi:10.1029/2000RG0001002002.

Sabra, K. G., P. Gerstoft, P. Roux, W. A. Kuperman, and M. C. Fehler (2005a), Extracting time-domain Green's function estimates from ambient seismic noise, Geophys. Res. Lett., 32, L03310, doi:10.1029/2004GL021862.

Sabra, K. G., P. Gerstoft, P. Roux, W. A. Kuperman, and M. C. Fehler (2005b), Surface wave tomography from microseisms in Southern California, Geophys. Res. Lett., 32, L14311, doi:10.1029/2005GL023155.

Sánchez-Sesma, F. J., and M. Campillo (2006), Retrieval of the Green's function from cross correlation: The canonical elastic problem, Bull. Seismol. Soc. Am., 96, 1182-1191.

Sánchez-Sesma, F. J., J. A. Pérez-Ruiz, M. Campillo, and F. Luzón (2006), Elastodynamic 2D Green function retrieval from cross-correlation: Canonical inclusion problem, Geophys. Res. Lett., 33, L13305, doi:10.1029/2006GL026454.

Schuster, G. T. (2009), Seismic Interferometry, Cambridge Univ. Press, New York. 
Shapiro, N. M., and M. Campillo (2004), Emergence of broadband Rayleigh waves from correlations of the ambient seismic noise, Geophys. Res. Lett., 31, L07614, doi:10.1029/2004GL019491.

Shapiro, N. M., M. Campillo, L. Stehly, and M. H. Ritzwoller (2005), High-resolution surface-wave tomography from ambient seismic noise, Science, 307, 1615-1618.

Stehly, L., M. Campillo, B. Froment, and R. L. Weaver (2008), Reconstructing Green's function by correlation of the coda of the correlation $\left(C^{3}\right)$ of ambient seismic noise, J. Geophys. Res., 113, B11306, doi:10.1029/2008JB005693.

Tanter, M., J.-F. Aubry, J. Gerber, J.-L. Thomas, and M. Fink (2001), Optimal focusing by spatio-temporal inverse filter. I. Basic principles, J. Acoust. Soc. Am., 110, 37-47.

Tsai, V. C. (2009), On establishing the accuracy of noise tomography travel-time measurements in a realistic medium, Geophys. J. Int., 178, $1555-1564$.

Tsai, V. C. (2011), Understanding the amplitudes of noise correlation measurements, J. Geophys. Res., 116, B09311, doi:10.1029/2011JB008483.

van Dalen, K. N., K. Wapenaar, and D. F. Halliday (2014), Surface wave retrieval in layered media using seismic interferometry by multidimensional deconvolution, Geophys. J. Int., 196, 230-242.

van der Neut, J., E. Ruigrok, D. Draganov, and K. Wapenaar (2010), Retrieving the Earth's reflection response by multi-dimensional deconvolution of ambient seismic noise, EAGE Extended Abstracts, pp. P406-1-P406-4.

van Wijk, K., T. D. Mikesell, V. Schulte-Pelkum, and J. Stachnik (2011), Estimating the Rayleigh-wave impulse response between seismic stations with the cross terms of the Green tensor, Geophys. Res. Lett., 38, L16301, doi:10.1029/2011GL047442.

Wapenaar, K. (2004), Retrieving the elastodynamic Green's function of an arbitrary inhomogeneous medium by cross correlation, Phys. Rev. Lett., 93, 254301.

Wapenaar, K., and J. Fokkema (2006), Green's function representations for seismic interferometry, Geophysics, 71, SI33-SI46.

Wapenaar, K., and J. van der Neut (2010), A representation for Green's function retrieval by multidimensional deconvolution, J. Acoust. Soc. Am., 128, EL366-EL371.

Wapenaar, K., E. Slob, R. Snieder, and A. Curtis (2010), Tutorial on seismic interferometry: Part 2 - Underlying theory and new advances, Geophysics, 75, 75A211-75A227.

Wapenaar, K., E. Ruigrok, J. van der Neut, and D. Draganov (2011a), Improved surface-wave retrieval from ambient seismic noise by multi-dimensional deconvolution, Geophys. Res. Lett., 38, L01313, doi:10.1029/2010GL045523.

Wapenaar, K., J. van der Neut, E. Ruigrok, D. Draganov, J. Hunziker, E. Slob, J. Thorbecke, and R. Snieder (2011b), Seismic interferometry by crosscorrelation and by multidimensional deconvolution: A systematic comparison, Geophys. J. Int., 185, 1335-1364.

Wapenaar, K., J. van der Neut, and J. Thorbecke (2012), On the relation between seismic interferometry and the simultaneous-source method, Geophys. Prospect., 60, 802-823.

Weaver, R. L., and O. I. Lobkis (2006), Diffuse fields in ultrasonics and seismology, Geophysics, 71, SI5-SI9.

Weemstra, C., L. Boschi, A. Goertz, and B. Artman (2013), Seismic attenuation from recordings of ambient noise, Geophysics, 78, Q1-Q14.

Yang, Y., M. H. Ritzwoller, F.-C. Lin, M. P. Moschetti, and N. M. Shapiro (2008), Structure of the crust and uppermost mantle beneath the western United States revealed by ambient noise and earthquake tomography, J. Geophys. Res., 113, B12310, doi:10.1029/2008JB005833.

Zhang, J., and X. Yang (2013), Extracting surface wave attenuation from seismic noise using correlation of the coda of correlation, J. Geophys. Res. Solid Earth, 118, 2191-2205, doi:10.1002/jgrb.50186. 